\title{
Secondary school students' misunderstandings of potential wells and tunneling
}

\author{
K. Krijtenburg-Lewerissa $\odot,{ }^{1}$ H. J. Pol, ${ }^{1}$ A. Brinkman, ${ }^{2}$ and W. R. van Joolingen ${ }^{3}$ \\ ${ }^{1}$ ELAN Institute for Teacher Training, University of Twente, 7500 AE Enschede, Netherlands \\ ${ }^{2}$ MESA + Institute for Nanotechnology, University of Twente, 7500 AE Enschede, Netherlands \\ ${ }^{3}$ Freudenthal Institute for Science and Mathematics Education, University of Utrecht, \\ 3508 AD Utrecht, Netherlands
}

(Received 21 June 2019; accepted 8 May 2020; published 1 June 2020)

\begin{abstract}
In order to investigate students' misunderstandings of potential wells and tunneling, a conceptual knowledge test was administered to Dutch secondary school students after they were taught about quantum mechanics. A frequency analysis of responses to the multiple choice questions $(n=98)$ and coding of the responses to the open-ended questions and explanations $(n=13)$ shows that Dutch secondary school students experience difficulties similar to those reported for undergraduate students. The students' underlying difficulties were analyzed using a typology of learning impediments. Results of this analysis show that students have difficulty connecting knowledge of potential wells and tunneling to their prior knowledge. Students mainly have creative and epistemological learning impediments, which cause eight incorrect synthetic models.
\end{abstract}

DOI: 10.1103/PhysRevPhysEducRes.16.010132

\section{INTRODUCTION}

Since quantum mechanics $(\mathrm{QM})$ plays a fundamental role in physics research and its applications, it has become part of the secondary school curriculum in many countries. Teaching QM at the secondary school level is challenging, because secondary school students have not learned to use the mathematical tools needed for a formal, mathematical approach to QM. Therefore, in secondary schools, QM needs to be taught at a conceptual level. Teaching QM at the secondary school level is also difficult because QM is fundamentally different from the classical physics that secondary school students have encountered [1]; daily life experiences are usually not associated with QM [2], which makes it counterintuitive. Students have the tendency to describe quantum phenomena deterministically [3], which conflicts with QM principles. Research has also shown that students tend to incorrectly generalize their prior knowledge of classical concepts [4]. For a good implementation of QM at secondary schools, knowledge of students' difficulties when learning QM is needed.

Research [5] has shown that there are QM topics that are taught in most international curricula, for instance, waveparticle duality and discrete energy levels. Most research into QM education at the secondary school level has focused on these topics [6]. However, less research has been conducted

Published by the American Physical Society under the terms of the Creative Commons Attribution 4.0 International license. Further distribution of this work must maintain attribution to the author(s) and the published article's title, journal citation, and DOI. regarding topics that are taught less frequently. This can be seen by the scarcity of research conducted on student understanding of the philosophical aspects of QM [2,7], or mathematical representations, for instance, the 1D infinite potential well, which is taught in the Netherlands [8]. A review [6] of the current knowledge of students' misunderstandings of QM showed that more research is needed into secondary school students' understanding of QM and their underlying difficulties, especially for students' understanding of the wave function, potential wells, and tunneling.

In this paper, we present our research into secondary students' (mis)understandings of the 1D infinite potential well and tunneling, which recently have become part of the Dutch secondary school physics curriculum [9]. To investigate Dutch students' understanding, a conceptual knowledge test was administered and the results were analyzed. To explore the underlying problems related to the observed misunderstandings, interviews were conducted.

\section{BACKGROUND}

In presenting our investigation of students' difficulties, first we will give an overview of existing research on students' difficulties regarding potential wells and tunneling. Additionally, we will discuss relevant conceptual change theories and the typology of learning impediments created by De Jong and Taber [10], which will be used to analyze the observed learning difficulties.

\section{A. Students' difficulties with potential wells and tunneling}

There has been research into students' understanding of potential wells [11] and tunneling [12-14], but mainly at 
the undergraduate level. This research has shown that students have difficulty understanding potential wells and tunneling, and often use classical reasoning. Research into other topics of QM has shown that students often describe the wave function as a classical particle moving over a sinusoidal trajectory [15-17]. This classical reasoning also causes students to describe potential wells as external objects, and to describe tunneling in terms of interaction of a particle with the barrier [12]. Singh, Belloni, and Christian observed that this misplaced classical thinking can be caused by a mix up of related concepts, and overgeneralization of previously learned concepts $[4,18]$. In our review [6] we assigned the different incorrect ideas found in literature to the two categories observed by Singh et al. (see Table I). That review of the current knowledge of students' misunderstandings showed that undergraduate students experience difficulty with learning QM, because they are not able to connect quantum behavior to the physical reality as they see it.

\section{B. Conceptual learning of QM}

QM is fundamentally different from classical mechanics, because at the quantum level objects behave like waves in certain circumstances, and like particles in other circumstances. In a theory of conceptual change for learning science concepts, Chi [19] specified three ontological categories: (i) entities, which, for example, have weight and occupy space, (ii) processes, which occur over time, and (iii) mental states, such as emotions or intentions. In classical mechanics a particle would belong to the "entities" category, and waves would belong to the "processes" category. However, in QM a quantum entity is both an entity and a process. This requires an ontological shift, in which the quantum entity belongs to a new ontological category, having both wave and particle properties [20]. To use this new ontology, students need the capability to move back and forth between wave and particle representations. Therefore, conceptual learning of QM is best approached not as the acquisition of static knowledge, but as a process of exploring, developing, and evaluating alternative explanatory models [21]. This should lead to a change in students' conceptual profile [22]. Students need to become aware of the limitations of different models and need to be capable of deciding which model or description is appropriate in a specific situation [23,24]. In order to understand the limitations of models and representations, students need to have the newly learned concepts correctly embedded in their existing knowledge structures. When these newly learned concepts are incorrectly integrated within students' prior knowledge, this leads to inconsistent and incorrect models, which are called synthetic models $[25,26]$. For example, Vosniadou, Vamvakoussi, and Skopeliti [26] describe synthetic models of the shape of the Earth, in which children have combined their idea of a flat Earth with the spherical model of the Earth. Many children create mixed, synthetic models, and, e.g., come up with a model of a flattened or hollow sphere. Since QM by its very nature is inconsistent with students' prior knowledge, these synthetic models are likely to be formed. When looking at Table I, the observed mix ups of related concepts and overgeneralizations of prior concepts are incorrect integrations of QM into students' prior knowledge, and hence are synthetic models. The misconception "wave functions describe a trajectory," for example, is a synthetic model in which students combine their prior ideas of particles and waves. Students learn that a QM entity shows both particle and wave behavior and therefore create a model in which a particle moves like a wave.

Students' difficulties and synthetic models can be classified using the typology of De Jong and Taber [10], which is shown in Fig. 1. This typology is based on the ideas of Ausubel [27]; deep learning will only happen when students can relate the new knowledge to their existing knowledge. In the first version of this typology [28], Taber explains that these different learning impediments are based on a consideration of what can go wrong when students try to include new knowledge into their existing framework.

TABLE I. Undergraduate students' incorrect ideas regarding wave functions, potentials, tunneling, and probability. Reprinted from K. Krijtenburg-Lewerissa et al. [6].

\begin{tabular}{|c|c|c|}
\hline & Overgeneralization of prior concepts & Mix up of related concepts \\
\hline \multirow[t]{3}{*}{ Wave functions and potentials } & Wave functions describe a trajectory & Change in amplitude causes change in energy \\
\hline & Potential wells are objects & $\begin{array}{l}\text { The amplitude or equilibrium of the wave function } \\
\text { is mixed up with energy }\end{array}$ \\
\hline & Height in potential graphs means position & $\begin{array}{l}\text { There is difficulty to distinguish between energy } \\
\text { and probability }\end{array}$ \\
\hline \multirow[t]{3}{*}{$\begin{array}{l}\text { Tunneling and } \\
\text { probability }\end{array}$} & $\begin{array}{l}\text { The amplitude of wave functions } \\
\text { is a measure of energy }\end{array}$ & $\begin{array}{l}\text { Only the tops of the waves, which overtop } \\
\text { the barrier, will pass }\end{array}$ \\
\hline & $\begin{array}{l}\text { Probability is described with classical } \\
\text { arguments (e.g., velocity) }\end{array}$ & $\begin{array}{l}\text { Part of the energy is reflected at a barrier } \\
\text { during tunneling }\end{array}$ \\
\hline & $\begin{array}{l}\text { Energy or effort is needed to tunnel } \\
\text { through a barrier }\end{array}$ & $\begin{array}{l}\text { A single particle is described as an ensemble } \\
\text { of particles }\end{array}$ \\
\hline
\end{tabular}




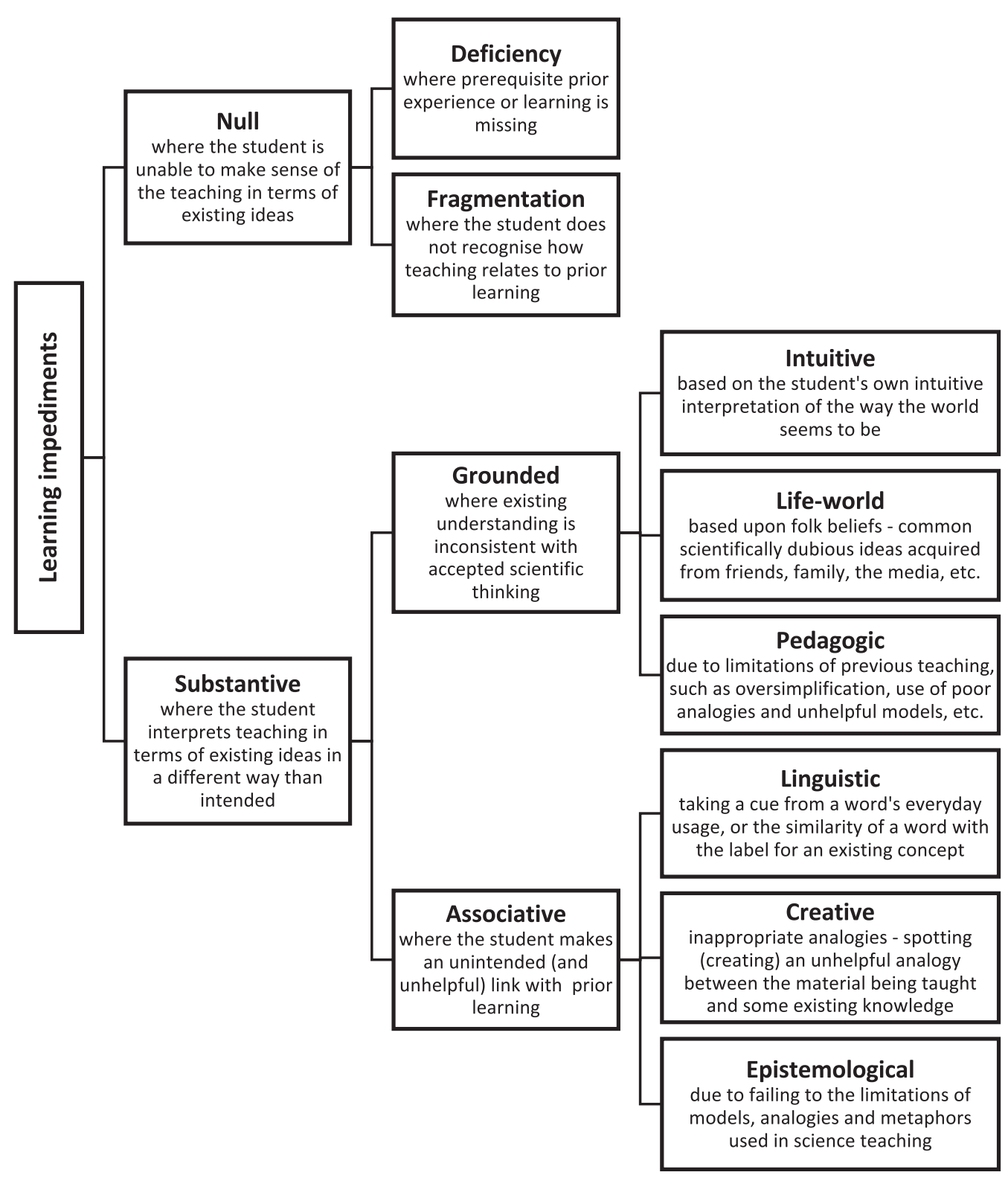

FIG. 1. The typology of learning impediments as presented by De Jong and Taber [10].

His main line of reasoning is similar to that of Vosniadou [25]. However, he does not only describe that there are incorrect models, but he also gives categories of underlying principles that impede deep learning. The typology is based on a consideration that students cannot make sense of a new concept when they lack prior knowledge, or do not recognize how new concepts relate to prior knowledge. They can also form alternative frameworks, which, for example, can be caused by their everyday experience, misinformation in society, teachers' misunderstanding or teachers' inability to present the new concept adequately. In a later study, Taber also observed that linguistic cues and students' epistemological assumptions play a role [29]. The typology presented
Fig. 1 was created during a study into difficulties that students encountered while learning particle theory.

Several of the misconceptions of Table I can be related to a learning impediment. The misconception "height in a potential graph means position," for example, can be related to a creative analogy to potential diagrams that students have encountered while learning classical physics. The misconception "potential wells are objects," can be related to the phrase "well" and therefore may be caused by a linguistic impediment. Since at first glance De Jong and Taber's typology can be related to QM misconceptions, this typology is a good starting point for analyzing students' understanding of QM. Additionally, this typology also 
helps to investigate the underlying principles that impede deep learning.

\section{METHOD}

In order to investigate students' understanding of the 1D infinite potential well and tunneling, a test was created. A review of existing tests on QM showed that there were only two multiple choice (MC) tests that partly addressed tunneling at a level that was appropriate for the Dutch secondary school level, the QMCI [30] and the QMCS [15]. No suitable test questions were found regarding the 1D infinite potential well. One of the questions on tunneling could be used at once, four other questions were slightly adapted. The questions were translated and verified by a content expert and two experts in physics pedagogy. The other four MC question were created by the authors, based on misconceptions found in their review [6]. To investigate not only the presence of known misconceptions, but also the underlying difficulties, the authors also created openended (OE) questions. These $\mathrm{OE}$ questions were mainly explanations; for each MC question, students were also asked to explain their choice. The $\mathrm{OE}$ questions were created to investigate if students were able to explain what the wave function and 1D potential well represented.

The test was given to 98 students during a physics class, at five different secondary schools after they were taught QM. The students were 17-18 years old and in their last year of pre-university education, The groups were chosen by convenience sampling. The test included the $\mathrm{QM}$ topics that are part of the secondary school physics curriculum in the Netherlands: (i) the wave character of light, (ii) wave-particle duality, (iii) the photoelectric effect, (iv) Heisenberg's uncertainty principle, (v) the $1 \mathrm{D}$ infinite potential well (i.e., the particle-in-a-box model), (vi) the hydrogen atom, and (vii) tunneling. In this article we discuss only the results of the 12 questions regarding the potential well and tunneling. Of these 12 questions, seven questions addressed the understanding of potential wells and wave functions, and five questions addressed the understanding of tunneling. The questions addressed the topics shown in Table II. See Supplemental Material [31] for the translated questions used in the test. The answers of the MC questions were used for a frequency analysis, while the explanations of the $\mathrm{MC}$ questions and responses to the $\mathrm{OE}$ questions were used for qualitative analysis.

For the qualitative analysis, 16 students were selected for interviews. This selection was based on an analysis of the MC questions. For the four schools participating in the interviews, we selected at least one student who had a low score, one student who had an intermediate score and one student who had a high score. Because of the absences, 13 of the selected students took part in the interviews. The interviews were conducted within six weeks after taking the test. During the interviews the students were asked to explain their answers to questions $2,3,5,8,9$, and 12 on the test. They were asked to $(\mathrm{Q} 2)$ explain what wave functions are and interpret a graph of a wave function, (Q5) explain the incorrect representation of the 1D infinite potential well, (Q3) compare two wave functions, (Q8) describe what happens with the energy of a particle during tunneling, and (Q9 and Q12) describe the influence of changing the width and height of the barrier on tunneling. For the 13 interviewed students, the interviews, explanations of $\mathrm{MC}$ questions during the test, and answers to $\mathrm{OE}$ questions were analyzed using open coding. Then, related codes were grouped together into categories of observed misunderstandings. To investigate the underlying problems, these categories of misunderstandings were analyzed using the typology of learning impediments of De Jong and Taber [10], leading to a more detailed framework for students' difficulties while learning QM. Finally, this framework was used to conduct a frequency analysis on the responses of the complete group of 98 students.

TABLE II. The content of the conceptual knowledge test.

\begin{tabular}{|c|c|c|c|c|}
\hline & Question & Question type & Source & Topic \\
\hline \multirow{7}{*}{$\begin{array}{l}\text { Potential wells and } \\
\text { wave functions }\end{array}$} & Q1 & $\mathrm{OE}$ & $\ldots$ & Explain the particle-in-a-box model \\
\hline & Q2 & $\mathrm{OE}$ & $\ldots$ & Interpret a wave function \\
\hline & Q3 & MC & $\cdots$ & Interpret a wave functions in terms of energy \\
\hline & Q4 & MC & $\cdots$ & $\begin{array}{l}\text { State what is a measure of the energy level in the } \\
\text { particle-in-a-box model }\end{array}$ \\
\hline & Q5 & $\mathrm{OE}$ & $\cdots$ & $\begin{array}{l}\text { Explain the incorrect representation of the } \\
\text { particle-in-a-box model }\end{array}$ \\
\hline & Q6 & $\mathrm{MC}$ & $\ldots$ & Interpret a wave functions in terms of probability distribution \\
\hline & Q7 & $\mathrm{MC}$ & QMCI Q9 & $\begin{array}{l}\text { State what property of a wave function is a measure } \\
\text { of the energy level }\end{array}$ \\
\hline \multirow[t]{5}{*}{ Tunneling } & Q8 & MC & QMCS Q7 & State how tunneling influences the energy level \\
\hline & Q9 & MC & QMCI Q2 & State how barrier height influences tunneling \\
\hline & Q10 & $\mathrm{MC}$ & QMCI Q6 & State how the energy level influences tunneling probability \\
\hline & Q11 & $\mathrm{MC}$ & $\ldots$ & Interpret how the potential barrier influences the energy \\
\hline & Q12 & $\mathrm{MC}$ & QMCI Q3 & State how barrier width influences tunneling \\
\hline
\end{tabular}




\section{QUANTITATIVE RESULTS}

The online conceptual knowledge test (see Supplemental Material [31]) was given to secondary school students after they had been taught QM $(n=98)$. The reliability of the MC part of the test, addressing potential wells and tunneling was determined with Cronbach's alpha: $\alpha=0.747$. Table III shows the percentage of students that answered the MC questions correctly. Since question 9 and 12 addressed the understanding of both probability and energy, these questions are presented as having two parts.

The results for the MC questions on potential wells and wave functions were analyzed to investigate the presence of known difficulties. Analysis of these questions showed that most students knew how the wave function relates to the position of the particle (Q6) and understood that wave functions with different frequencies must have different energy levels (Q3). However, when students were asked what defines the energy level (Q4, Q7), 24\% of the students believed that the amplitude of the wave function influences the energy level. The questions addressing students' understanding of tunneling showed that approximately $50 \%$ of the students believed that energy decreases after tunneling, a difficulty that has been previously reported for undergraduate and graduate students $[11,12,32]$. However, secondary school students seem to have more difficulty understanding tunneling. Question 8 was answered correctly by only $39 \%$ of the students, which is significantly less that the results for this question in the QMCS [15], where $75 \%$ of the graduate students answered this question correctly. What also stood out was the difference between students' ideas concerning the influence of the width and height of the barrier on the energy level; $20 \%$ of the students believed that the height of the barrier influences the energy level, whereas $45 \%$ of the students believed that the width of the barrier influences the energy level. This is in line with the results of McKagan and Wieman [33], who found that $19 \%$ of the graduate students believed that the width influenced the energy of the particle, whereas $11 \%$ believed that the height of the barrier influences the energy.

The results of the MC questions give an overview of incorrect knowledge, but they give no information about the underlying ideas that cause students' difficulties. Therefore, the incorrect beliefs found in this study were examined more thoroughly in the qualitative analysis of the

TABLE III. Results MC questions on potential wells and tunneling $(n=98)$.

\begin{tabular}{|c|c|c|c|c|}
\hline Topic & Question & Subtopic & $\begin{array}{l}\text { Students with a } \\
\text { correct answer }(\%)\end{array}$ & Remarks \\
\hline \multirow[t]{4}{*}{$\begin{array}{l}\text { 1D infinite potential well } \\
\text { and wave functions }\end{array}$} & Q3 & $\begin{array}{l}\text { Frequency is related to the } \\
\text { energy level }\end{array}$ & 80 & $\begin{array}{l}10 \% \text { chose the option that wave } \\
\text { functions with different } \\
\text { frequencies have the } \\
\text { same energy level }\end{array}$ \\
\hline & Q4 & $\begin{array}{l}\text { Number of nodes of the standing } \\
\text { wave is related to the energy level }\end{array}$ & 56 & $\begin{array}{l}24 \% \text { chose amplitude, } \\
\text { and } 12 \% \text { the area under the } \\
\text { curve as a measure of the } \\
\text { energy level }\end{array}$ \\
\hline & Q6 & $|\Psi|^{2}$ is the probability distribution & 93 & \\
\hline & Q7 & Frequency is related to the energy level & 63 & $\begin{array}{l}24 \% \text { chose amplitude as a } \\
\text { measure of the energy level }\end{array}$ \\
\hline \multirow[t]{7}{*}{ Tunneling } & Q8 & $\begin{array}{l}\text { Total energy remains equal after } \\
\text { tunneling }\end{array}$ & 39 & $\begin{array}{l}51 \% \text { chose the option that } \\
\text { the energy after tunneling } \\
\text { is decreased }\end{array}$ \\
\hline & \multirow[t]{2}{*}{ Q9 } & $\begin{array}{l}\text { Increasing the barrier height decreases } \\
\text { the probability of tunneling }\end{array}$ & 64 & \\
\hline & & $\begin{array}{l}\text { Increasing the barrier height does not } \\
\text { influence the particle's energy level }\end{array}$ & 80 & \\
\hline & Q10 & $\begin{array}{l}\text { Probability of tunneling does not solely } \\
\text { depend on the particle's and barrier's } \\
\text { energy levels }\end{array}$ & 76 & $\begin{array}{l}12 \% \text { chose the option that } \\
\text { the probability of tunneling } \\
\text { is } 0 \text {, and } 11 \% \text { that it is } 0.5\end{array}$ \\
\hline & Q11 & $\begin{array}{l}\text { Energy remains equal after } \\
\text { tunneling (visual) }\end{array}$ & 38 & $\begin{array}{l}48 \% \text { chose the option that the } \\
\text { energy after tunneling is } \\
\text { decreased }\end{array}$ \\
\hline & \multirow[t]{2}{*}{ Q12 } & $\begin{array}{l}\text { Increasing the barrier width decreases } \\
\text { the probability of tunneling }\end{array}$ & 74 & \\
\hline & & $\begin{array}{l}\text { Increasing the barrier width does } \\
\text { not influence the particle's } \\
\text { energy level }\end{array}$ & 55 & \\
\hline
\end{tabular}


explanations of the $\mathrm{MC}$ questions, the responses to the $\mathrm{OE}$ questions, and the interview transcripts.

\section{QUALITATIVE RESULTS}

In order to investigate if there are more difficulties, and to find underlying problems, the explanations, OE questions, and interviews were analyzed of the 13 selected students. First, open coding was used to analyze these students' responses. This led to 299 codes, which described students' correct and incorrect lines of reasoning. After merging overlapping codes, 160 codes remained, of which 77 described incorrect ideas. These 77 codes were analyzed and grouped into the 12 codes shown in Table IV. The areas of difficulty found for the Dutch secondary school students are similar to the undergraduate students' incorrect ideas presented in Table I. Most of these incorrect ideas can be considered to be synthetic models in which students have created incorrect links to prior knowledge. Table V shows which students used these incorrect ideas in their reasoning during the test and in the interviews. In this table, it can be

TABLE IV. The incorrect ideas on potential wells and tunneling observed in the explanations of the MC questions, the responses to the OE questions, and in the interviews $(n=13)$.

\begin{tabular}{ll}
\hline \hline Topic & \multicolumn{1}{c}{ Subtopic } \\
\hline $\begin{array}{l}\text { 1D infinite potential well } \\
\text { and wave functions }\end{array}$ & The model gives information about the particle's height \\
& The well is a physical object \\
& The particle has classical wave properties \\
& The well is linked to resistance \\
& The equilibrium is a measure of the energy level \\
& The amplitude is a measure of the energy level \\
& Incorrect use of amplitude and wavelength in energy equations \\
& The particle loses energy during tunneling \\
& After tunneling the particles energy is increased \\
& The particles energy needs to exceed a threshold for tunneling \\
Tunneling & Either the width or the height of the barrier solely influences tunneling \\
& Incorrect reasoning with frequency or amplitude of the wave function \\
\hline \hline
\end{tabular}

TABLE V. The distribution of the incorrect ideas over the 13 selected students. S1-S4 scored high, S5-S9 intermediate, and S10-S13 low on the MC questions.

\begin{tabular}{|c|c|c|c|c|c|c|c|c|c|c|c|c|c|c|c|}
\hline & & \multicolumn{4}{|c|}{ High scoring } & \multicolumn{5}{|c|}{ Intermediate scoring } & \multicolumn{5}{|c|}{ Low scoring } \\
\hline & & S1 & $\mathrm{S} 2$ & S3 & S4 & S5 & S6 & S7 & S8 & S9 & S10 & S11 & $\mathrm{S} 12$ & S13 & Totals \\
\hline \multirow{7}{*}{$\begin{array}{l}\text { 1D potential wells } \\
\text { and wave } \\
\text { functions }\end{array}$} & $\begin{array}{l}\text { The model gives information } \\
\text { about the particles height }\end{array}$ & & & $\bullet$ & $\bullet$ & & - & & & & & $\bullet$ & $\bullet$ & - & 6 \\
\hline & The well is a physical object & & & & & $\bullet$ & & & & & & & $\bullet$ & & 2 \\
\hline & $\begin{array}{l}\text { The particle has classical } \\
\text { wave properties }\end{array}$ & & $\bullet$ & & & & • & & $\bullet$ & • & - & $\bullet$ & $\bullet$ & • & 8 \\
\hline & The well is linked to resistance & & & & & & & & & & & & - & & 1 \\
\hline & $\begin{array}{l}\text { The equilibrium is a measure } \\
\text { for the energy level }\end{array}$ & $\bullet$ & & $\bullet$ & & & - & $\bullet$ & $\bullet$ & & $\bullet$ & & & & 6 \\
\hline & $\begin{array}{l}\text { The amplitude is a measure } \\
\text { of the energy level }\end{array}$ & $\bullet$ & - & $\bullet$ & & & & & & $\bullet$ & $\bullet$ & • & $\bullet$ & - & 8 \\
\hline & $\begin{array}{l}\text { Incorrect use of amplitude and } \\
\text { wavelength in energy equations }\end{array}$ & & & & & & & $\bullet$ & & & & & & $\bullet$ & 2 \\
\hline \multirow[t]{6}{*}{ Tunneling } & $\begin{array}{l}\text { The particle loses energy during } \\
\text { tunneling }\end{array}$ & & - & & $\bullet$ & $\bullet$ & $\bullet$ & & $\bullet$ & $\bullet$ & $\bullet$ & $\bullet$ & • & - & 10 \\
\hline & $\begin{array}{l}\text { After tunneling the particles } \\
\text { energy is increased }\end{array}$ & & & & & & & $\bullet$ & $\bullet$ & & & $\bullet$ & & & 3 \\
\hline & $\begin{array}{l}\text { The particles energy needs to exceed } \\
\text { a threshold for tunneling }\end{array}$ & $\bullet$ & & $\bullet$ & & $\bullet$ & • & - & $\bullet$ & & & $\bullet$ & & & 7 \\
\hline & $\begin{array}{l}\text { Either the width or the height of the } \\
\text { barrier solely influences tunneling }\end{array}$ & & $\bullet$ & $\bullet$ & & & • & & & $\bullet$ & $\bullet$ & & $\bullet$ & & 6 \\
\hline & $\begin{array}{l}\text { Incorrect reasoning with frequency } \\
\text { or amplitude of the wave function }\end{array}$ & & & & & & & & & $\bullet$ & $\bullet$ & & & & 2 \\
\hline & Totals & 3 & 4 & 5 & 2 & 3 & 6 & 4 & 5 & 5 & 6 & 6 & 7 & 5 & 61 \\
\hline
\end{tabular}


seen that high scoring students can still use incorrect explanations. In the interviews the students were asked to explain questions $2,3,5,8,9$, and 12 of the test, to probe more deeply the underlying problems behind the incorrect views and synthetic models.

In the following sections, a more detailed description of students' incorrect views will be given, together with an analysis of the types of learning impediments that play a role. The analysis is based on the typology of learning impediments presented in Fig. 1. In this typology, students can have a "null learning impediment" when they have not been able to connect the new knowledge to their own existing knowledge. Incorrect reasoning based on lack of knowledge will be considered to be a deficiency learning impediment. When students' reasoning shows that they do not incorporate the new knowledge into their existing knowledge, and therefore do not know how to describe or use new knowledge, this will be considered to be a fragmentation learning impediment. Students can also have a "substantive learning impediment" when they connect the new knowledge incorrectly to prior knowledge, and come up with a synthetic model. Reasoning based on students' own intuitive interpretation of the world will be considered to be an intuitive learning impediment, and reasoning based on beliefs acquired from friends, family and media, will be considered to be a life-world learning impediment. Students can also interpret new knowledge incorrectly because of limitations of previous teaching, this will be considered to be a pedagogic learning impediment. When students make an unintended link because of the similarity of a word with an existing concept, this will be considered to be a linguistic learning impediment. When students' reasoning is based on correct, but inappropriate, previously learned models in related contexts, this will be considered to be an epistemological learning impediment, when students incorrectly mix-up different physical representations and this is not caused by limitations of models, this will be considered to be a creative learning impediment.

\section{A. The 1D potential well and wave functions}

To gain understanding of students' difficulties, the quotes from $\mathrm{MC}$ explanations, $\mathrm{OE}$ questions, and the interviews belonging to an incorrect idea were compared, grouped, and categorized within the framework of learning impediments (Fig. 1). In this section we describe the students' responses regarding the 1D potential well and wave functions. We also illustrate these descriptions with quotes, and explain to which learning impediments these responses correspond.

\section{The model gives information about the particle's height}

When confronted with the incorrect wave-energy representation of Q5, six students stated that the straight horizontal lines represent the height of the particle. When looking deeper into these students' reasoning, several of them seem to have difficulty connecting the learned concepts with their previously learned models and representations. As a result, four students used a previously learned semiclassical model to explain the 1D potential well:
S6: ... these are electrons in different shells ... closer to the nucleus the energy is lower.
S11: I would say it is higher, but I do not know if I can explain it with physics. I'd better think in terms of chemistry... I would look at those [refers to shells which were mentioned earlier]...

This use of an inappropriate model can be seen as an epistemological learning impediment.

The other two students tried to explain the 1D potential well by mixing it with prior knowledge of classical waves:

S12: As I learned from physics... isn't that the average that the particle moves around? So it is the average distance from the nucleus.

S13: When the particle gets higher, it goes faster and creates more standing waves.

This mix up of different representations can be seen as a creative learning impediment.

\section{The well is a physical object}

When the students were asked what the vertical lines in the 1D potential well represented, two students tried to explain the vertical lines, using classical, deterministic thinking. One of these students could only explain the figure within the analogy of a physical well:

\section{S12: The edges are the walls of the well?}

This can be seen as both an overgeneralization of an analogy (epistemological) and a problem of wording (linguistic). Because of this students' specific choice of words, this explanation was interpreted as a linguistic learning impediment, which is caused by the use of the word "well."

The other student uses her knowledge that the potential is a simplified atomic model, describing the vertical lines as boundaries of an atom.

S5: ...those are the boundaries, the radius of the atom.

But this deterministic reasoning also led to incorrect ideas:

\section{S5: The bottom represents the nucleus of the atom.}

These examples show that this student linked the potential well to the atomic model, but took this too far. This can be seen as an epistemological learning impediment.

Only three students related the vertical line to the potential energy of the system. Two students did not know 
what the figure represented and could not relate the energy diagram to their prior knowledge. Still, seven of the students were aware that the infinite potential determined the possible positions of the electron.

\section{The particle has classical wave properties}

When explaining the 1D infinite potential well, students were also reasoning about the wave character of the electron within the well. Most of the students related the wave function to prior knowledge of classical waves, but because the electron also behaves like a particle, this knowledge of waves was combined with a deterministic description of the electron's path. This led to a mixed model in which the electron vibrates or moves along a sinusoidal path:

S8: that is the equilibrium which it moves around.

S13: It has to do with a vibration or how fast it moves.

S11: It moves along these lines.

S12: The particle moves like a wave and has a tone.

This mix up of different models can be seen as a creative learning impediment. However, these creative, mixed, and incorrect models seem to be caused by the inability of students to incorporate the correct, nondeterministic representation in their thinking:

S12: I find that a difficult question... because I think this line represents a probability, our teacher stated that yesterday. And the particle is there, not straight, but a little bit as a wave... but, maybe it moves like that. Its position however, is completely random.'

This student had been taught that the wave function gives information about the probability distribution of the electron. Since this student does not know how the probability distribution relates to his prior knowledge, he keeps thinking in terms of movement and position, which can be seen as a fragmentation learning impediment.

Other students showed that their ideas were incomplete, when they were asked to elucidate their descriptions:

S9: I think this one moves more.

I: What does that mean?

S9: I don't know... position?

I: You say it moves more, or more often.

S9: More, so it has more energy.

I: And...

S9: So it has a... I don't know.

I: I don't understand what you mean by "movement"

S13: That this is the highest velocity it can have, but that... that is not true.

These students could not relate the wave behavior of electrons to their prior, classical, knowledge, which is a fragmentation learning impediment.

\section{The well is linked to resistance}

When the students were asked to explain the potential well, one student linked it to resistance:

S12: ...the particle cannot escape. This way the concept of "resistance" can be clarified.

In other parts of the interview, the student talked about collisions. Therefore, it is likely that the word resistance refers to forces or interactions working on the particle. This mix up of resistance, forces, and potential energy can be seen as a creative learning impediment.

\section{The equilibrium is a measure of the energy level}

When students were asked what is a measure of the energy level in the 1D potential well, four students stated that the equilibrium of the wave function represents the energy level, and not the wavelength:

S3: I assume that the $x$ axis [student points to equilibrium] lies at $y=0$, so it has a low energy level.

S10: The higher the equilibrium, the higher the particle's potential energy

This is a mix up of two representations (the equilibrium and the energy level), which can be seen as a creative learning impediment.

However, the second statement is correct for representations similar to Fig. 2. Two other students specifically referred to a representation as shown in Fig. 2. During the interviews these students were confused, because in Q3 of the test, the wave functions with different energy levels both intercepted $y=0$.

S1: This [student points to the equilibrium] should be drawn higher.

Since both students specifically used the incorrect 1D potential well representation in their reasoning, this can be

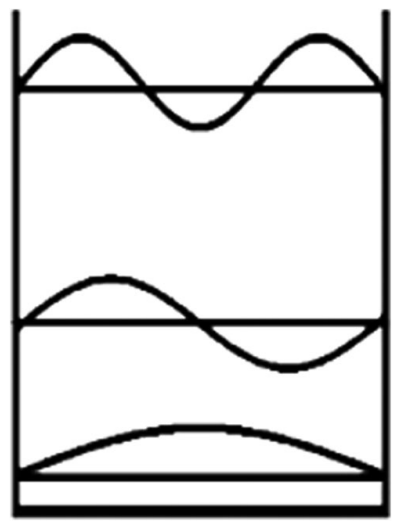

FIG. 2. The incorrect $1 \mathrm{D}$ potential well representation, which simultaneously shows wave functions and energy levels. 


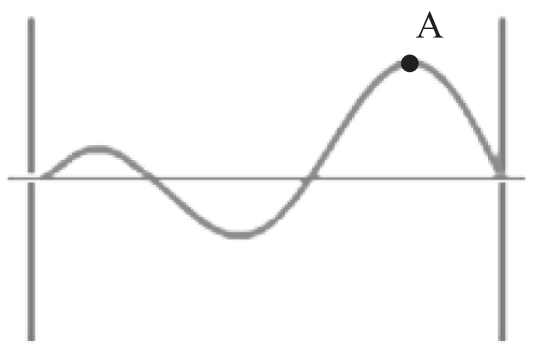

FIG. 3. A standing wave within the 1D potential well.

seen as a pedagogic learning impediment, resulting from the use of this representation in text books. This shows that the pedagogic learning impediment can lead to a creative learning impediment.

\section{The amplitude is a measure of the energy level}

When students were asked to explain the figures belonging to Q2 and Q3, students linked the displacement or amplitude to the energy level:

S10: The vertical axis is the energy level. The amplitude of both wave functions is equal, so the energy level is equal.

S11: The electron is moving and gets a higher energy, because the second part of the sine is higher than the first.

S12: The bigger the displacement, the higher the particle's energy level.

Two students mixed up the wave representation with a deterministic atomic model, and linked the sinusoidal path to the movement between two different energy states or shells. While pointing at point A in Fig. 3, one of these students said:

\section{S2: ...the excited state is located at this position...}

When we asked why the student linked this to an excited state, the student stated that she believed so, because the figure had to do with the particle's position.

These results show that these incorrect ideas are creative learning impediments. The idea that the $y$ axis represents both position and energy can be caused by the incorrect potential well representation of Fig. 2 and can be seen as a pedagogic learning impediment as well.

\section{Incorrect use of amplitude and wavelength in energy equations}

Two students showed they did not understand the relation between energy equations they had learned and the potential well. Both students used an incorrect quantity as a parameter in an energy equation. This can be considered as a fragmentation learning impediment, since these students did not see how the energy equations relate to the potential well model.

\section{B. Tunneling}

To gain understanding of students' difficulties regarding tunneling, the quotes from the test and the interviews were compared, grouped, and categorized within the framework of learning impediments (Fig. 1). In this section we describe the students' responses regarding tunneling. We also illustrate these descriptions with quotes, and explain to which learning impediments these responses correspond.

\section{The particle loses energy during tunneling}

When the students were asked to compare the energy before and after tunneling, 10 students stated that the energy after tunneling is lower, because the particle loses energy. Three students referred to some sort of interaction:

S12: The particle loses energy, because of collisions.

S5: ...it is harder for the particle to get through the barrier.

Six students described the path of the particle during tunneling:

S11: The particle has to cover a longer distance

S8: When the barrier gets wider, the particle has to bridge a longer distance.

These students used the classical description of a particle, to describe a nondeterministic phenomenon. Since these students used a correct model in the wrong context, these approaches can be regarded as epistemological learning impediments.

One student mixed up the potential well and the barrier:

S13: Because it keeps moving back and forward [points to the barrier] ... but it will tunnel through...

This can be considered a creative learning impediment, in which two models are being mixed up and used to create a new, incorrect model.

\section{After tunneling the particle's energy is increased}

Three students stated that the particle's energy after tunneling is higher. One student thought that the particle must have a higher final energy level, to be able to stay on that side of the barrier:

S8: The particle's energy must be larger after tunneling, otherwise it would fall back.

The original Dutch phrase translated as "fall back" is a physical phrase that is directly related to electron excitation. This student mixes up the energy before and after tunneling with the quantized energy levels before and after 
excitation, which are substantially different contexts. Therefore, this explanation can be regarded as a mix up with quantized energy levels, which is a creative learning impediment.

Two of these students also believe that energy is lost during tunneling, one of them explained that he thinks that the energy initially becomes larger and then decreases:

S7: It [the particle] needs a lot of energy for tunneling and afterwards the energy decreases, but stays higher than the energy at the beginning...

These students described this process in a classical way, which can be seen as an epistemological learning impediment.

\section{The particle's energy needs to exceed a threshold for tunneling}

Three students who answered Q9 correctly, explained their answer by saying that the particle needs more energy when the barrier is higher.

S5: ... the particle needs to have more energy to get across.

These students knew that the height of the barrier relates to the tunneling probability, but still reasoned deterministically. This deterministic reasoning can be seen as an epistemological learning impediment.

When students were asked to choose the tunneling probability when the particle's energy level is half the barrier's energy level, two students stated that the particle's energy needs to be higher than the barriers energy level:

S6: The particle's energy is only half of the barriers energy, so it can never go through the barrier, because the barrier is too big.

The other two students thought that there is a specific amount of energy needed to go through the barrier. When we asked what will happen if the particle's energy level is higher, but still lower than that of the barrier, one of them said:

S11: I just think it needs a specific energy to go through. If it has an energy higher than that, maybe it will go through a little bit easier.

These incorrect ideas are related to students' knowledge of energy and barriers in classical systems, so this can be seen as an epistemological learning impediment.

\section{Either the width or the height of the barrier solely influences tunneling}

Five students believed that only the width of the barrier influences tunneling. When asked why, these students reasoned deterministically, which can be seen as an epistemological learning impediment:

S10: The particle doesn't go over the barrier, but through it. When the barrier becomes higher, the distance that the particle has to bridge doesn't get longer.

S6: When the barrier becomes wider, the particle has more time to lose energy.

S12: The height doesn't increase the resistance, only the width does.

Two students stated that only the height of the barrier influences tunneling. These students reasoned that only the difference between the energy level of the particle and the barrier influences tunneling;

S2: The energy difference stays the same ... so that doesn't make a difference.

These students only reasoned with energy and lacked knowledge of the influence of the barrier width on the wave functions, which is helpful in understanding tunneling. Since the influence of the barrier on the wave function is not part of the curriculum, this can be seen as a pedagogic learning impediment.

\section{Incorrect reasoning with frequency or amplitude of the wave function}

While explaining tunneling, several students used the difference between the wave function on both sides of the barrier. Some students had difficulties interpreting the wave function. One student linked the energy level to the amplitude of the wave function, which led to the believe that the energy is lower after tunneling. In accordance with section A, this can be seen as a creative learning impediment. Another student falsely stated that the frequency of the wave function is higher after tunneling and therefore concluded that the energy after tunneling is higher. This is an error in recollection of the shape of the wave function, not in understanding.

\section{Overview of observed learning impediments}

In the previous paragraphs we identified several observed learning impediments. Table VI shows the learning impediments that were found for each misunderstanding. We did not observe deficiency, intuitive, and life-world learning impediments, which is probably caused by unfamiliarity with, and the abstract nature of, potential wells and tunneling. There were three types of fragmentation learning impediments: students could not relate nondeterministic concepts (probability and the wave function) to their deterministic worldview, did not know how the energy equations related to the different representations, and could not relate energy diagrams correctly to their prior 
TABLE VI. The learning impediments found in the responses of the selected students.

\begin{tabular}{|c|c|c|c|c|c|c|}
\hline & & Fragmentation & Pedagogic & Linguistic & Creative & Epistemological \\
\hline \multirow{7}{*}{$\begin{array}{l}\text { 1D potential wells } \\
\text { and wave } \\
\text { functions }\end{array}$} & $\begin{array}{l}\text { The model gives information } \\
\text { about the particle's height }\end{array}$ & & & & • & • \\
\hline & The well is a physical object & & & $\bullet$ & & $\bullet$ \\
\hline & $\begin{array}{l}\text { The particle has classical } \\
\text { wave properties }\end{array}$ & $\bullet$ & & & $\bullet$ & \\
\hline & The well is linked to resistance & & & & • & \\
\hline & $\begin{array}{l}\text { The equilibrium is a measure } \\
\text { for the energy level }\end{array}$ & & • & & • & \\
\hline & $\begin{array}{l}\text { The amplitude is a measure } \\
\text { of the energy level }\end{array}$ & & • & & • & \\
\hline & $\begin{array}{l}\text { Incorrect use of amplitude and } \\
\text { wavelength in energy equations }\end{array}$ & • & & & & \\
\hline \multirow[t]{5}{*}{ Tunneling } & The particle loses energy during tunneling & & & & • & $\bullet$ \\
\hline & $\begin{array}{l}\text { After tunneling the particles energy } \\
\text { is increased }\end{array}$ & & & & • & • \\
\hline & $\begin{array}{l}\text { The particles energy needs to exceed a } \\
\text { threshold for tunneling }\end{array}$ & & & & & $\bullet$ \\
\hline & $\begin{array}{l}\text { Either the width or the height of the } \\
\text { barrier solely influences tunneling }\end{array}$ & & - & & & $\bullet$ \\
\hline & $\begin{array}{l}\text { Incorrect reasoning with frequency or } \\
\text { amplitude of the wave function }\end{array}$ & & & & • & \\
\hline
\end{tabular}

knowledge. Two types of pedagogic learning impediments were found: students believed that the $y$ axis of the 1D infinite potential well represented both position and energy, and did not know what happens with the wave function during tunneling. Because flaws in teaching can lead to other learning impediments, sometimes pedagogic learning impediments coexisted with other learning impediments. In addition, the questions in the tests, and explanations in Dutch text books often used deterministic phrases, such as "tunneling through a barrier." This may have promoted students' deterministic reasoning and may be seen as a pedagogic learning impediment. One linguistic learning impediment was found: one student interpreted the 1D infinite potential well literally. The observed creative learning impediments can be divided into four types. Students mixed up the quantum particle's wave behavior with properties of classical waves, the 1D infinite potential well and the barrier, the amplitude of the wave function with energy states, and the potential well with other classical concepts. Three types of epistemological learning impediments were found: students used inappropriate atomic models, reasoned classically in terms of energy, and reasoned deterministically. A schematic overview of the observed learning impediments can be found in Fig. 4, illustrated with students' quotes.

\section{Analysis open-ended questions and explanations $(n=98)$}

Finally, the responses to the explanations of the MC questions and the responses to the $\mathrm{OE}$ questions and explanations were analyzed for the complete sample, using the learning impediments in Fig. 4. The coding scheme, that was created for this analysis, is based on the typologies of Fig. 1 and the types of impediments that were presented in Fig. 4. When students did not incorporate the new knowledge to their existing knowledge, and therefore did not know how to describe or use new knowledge, this was considered to be a fragmentation learning impediment, three types of fragmentation were coded:

F1 Nondeterminism: Students do not know how to describe probability distribution.

F2 Equations and relations: Students do not know how to use equations and relations correctly.

F3 Energy diagrams: Students do not know that the potential well and barrier represent energy.

When students interpreted new knowledge incorrectly because of limitations of previous teaching, this was considered to be a pedagogic learning impediment; the following types of pedagogic impediments were coded:

P1 Mixed representations: Students think that the $y$ axis in a potential well represents the amplitude.

P2 Wave functions during tunneling: Students do not use the wave function in order to describe the probability of tunneling.

When students make an unintended link because of the similarity of a word with an existing concept, this was considered to be a linguistic impediment:

L1 "well"- analogy: Students refer to properties of a physical well.

When students make an unintended link with prior knowledge and create an analogy or a mix up that is not caused by limitations of models, this was considered to be a 


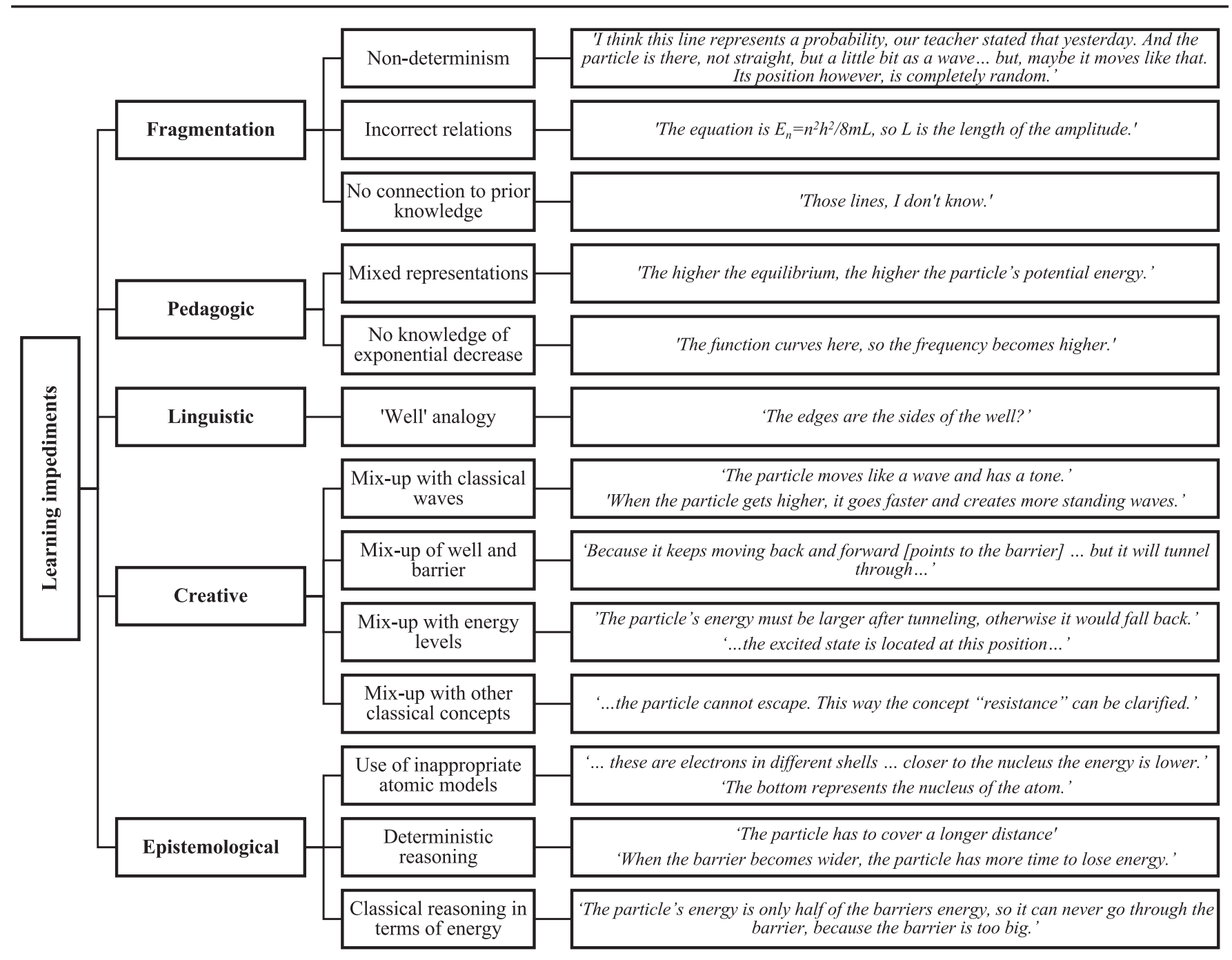

FIG. 4. An overview of the observed learning impediments.

creative learning impediment; four types of creative impediments were coded:

C1 Mix up with classical waves: Students mix up the particle's wave behavior with properties of classical waves, resulting in a mix of wave and particle behavior, e.g., a sinusoidal path or a vibrating particle.

C2 Mix up of well and barrier: Students mix up properties of a bounded and a free particle.

C3 Mix up with energy level: Students mix up the equilibrium or amplitude of the wave function with an energy level, or describe tunneling in terms of energy levels.

C4 Mix up with other classical concepts: Students mix up the potential well with other (semi-) classical concepts.

When students make an unintended link that was caused by limitations of previously learned models (i.e., overgeneralization), this was considered to be an epistemological learning impediment. Three types of overgeneralization were coded:
E1 Use of inappropriate atomic models: Students use previously learned atomic models to describe the infinite potential well and energy levels.

E2 Deterministic reasoning (movement): Students describe tunneling deterministically in terms of position, movement, and time.

E3 Classical reasoning (energy): Students describe tunneling deterministically and stated that a certain amount of energy was needed, or that the barrier absorbs energy.

The difference between the creative and epistemological learning impediments in this context is small and somewhat indefinite. We chose to code mix ups with parts of prior classical knowledge (such as the sine wave and vibration) leading to combined models (a particle following a sinusoidal path) as creative. The epistemological learning impediments were impediments that were based on using a single model or perspective (atomic models, determinism) in an inappropriate situation, without a mix up of different 
Synthetic model

Visual representation and quote

1
the potential well is a physical well
(L1)

'The edges are the sides of the well?'(S12)

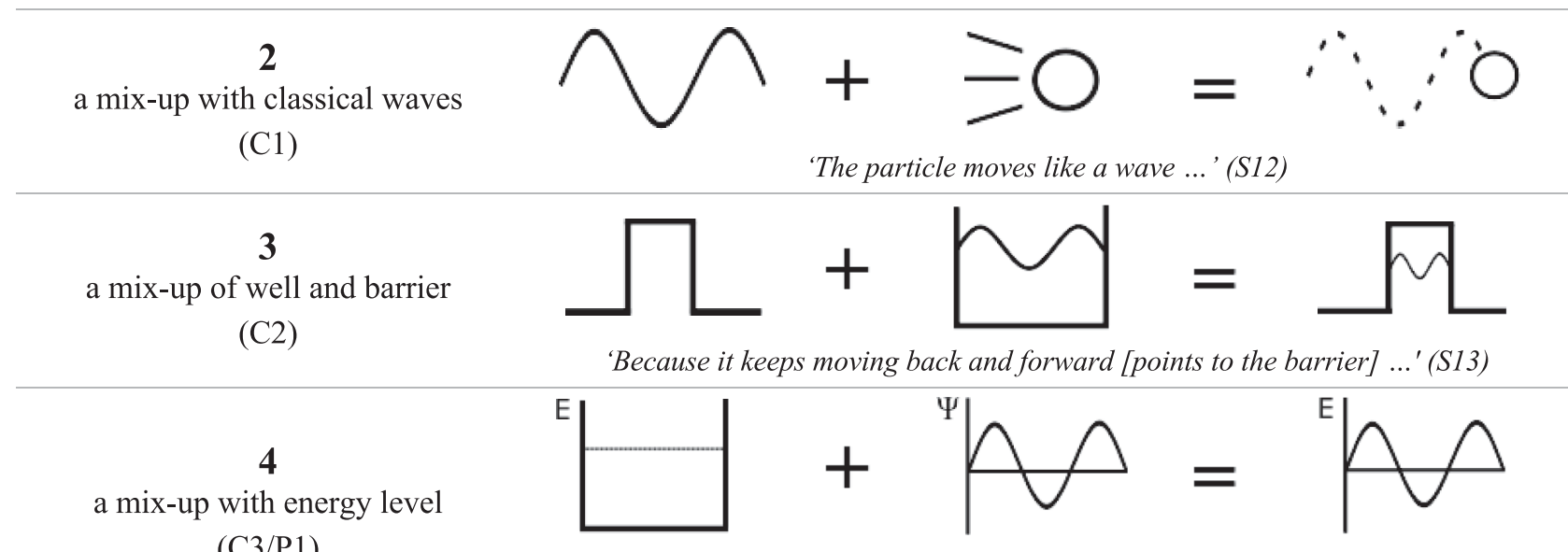

(C3/P1)

The electron is moving and gets a higher energy, because the second part of the sine is higher than the first.' (S11)

5

a mix-up with other classical concepts

(C4)

6

the use of inappropriate atomic models

(E1)

$\mathbf{6}$
the use of inappropriate atomic
models
$(\mathrm{E} 1)$
$\mathbf{7}$
deterministic reasoning in terms of
movement
$(\mathrm{E} 2)$
$\mathbf{8}$
classical reasoning in terms of
energy

(E3)
'This way the concept "resistance" can be clarified.' (S12) "... waves with a smaller wavelength are further from the cell wall' (S37)
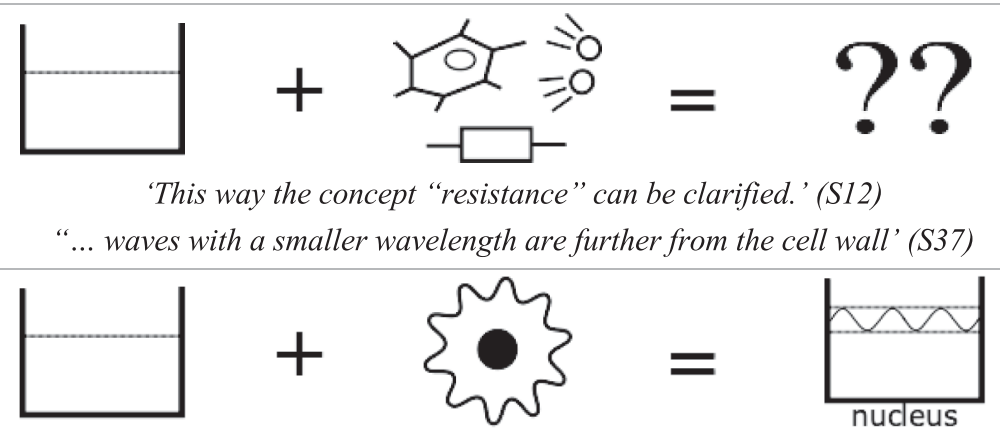

'The nodes and antinodes show the shape of the shell in which the particle is located' (S59)

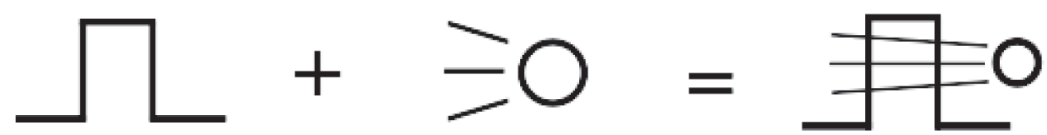

'The particle doesn't go over the barrier, but through it.' (S10)

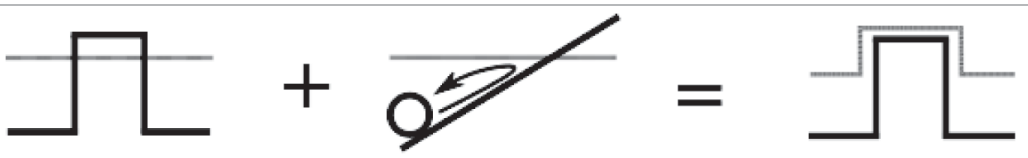

'... the particle needs to have more energy to get across.' (S5)

FIG. 5. An overview and a visual representation of the eight synthetic models found in this study.

models or perspectives. Since students did not learn about the wave function in the context of tunneling, this led to fewer mix ups for tunneling.

Finally, the codes used in this analysis are not mutually exclusive. For example, the pedagogic learning impediment that the $y$ axis of a potential well represents the amplitude of the wave function (P1) can lead to a mix up of the amplitude of the energy level (C3). Also, the linguistic learning impediment (L1) can lead to deterministic reasoning, since the words students know are all based on their classical experiences and can lead to a classical description of quantum phenomena.

The coding was checked for interrater reliability, Cohen's kappa was 0.67. Table VII shows the occurrence 
TABLE VII. The learning impediments found in the explanations, OE questions, and interviews.

\begin{tabular}{lclc}
\hline \hline Learning impediment & Code & \multicolumn{1}{c}{ Specific difficulty } & Students with this difficulty (\%) \\
\hline Fragmentation & F1 & Nondeterminism & 7 \\
& F2 & Incorrect relations & 6 \\
Pedagogic & F3 & No connection to prior knowledge & 8 \\
& P1 & Mixed representation & 20 \\
Linguistic & P2 & No knowledge of exponential decrease & 7 \\
Creative & L1 & "Well" analogy & 1 \\
& C1 & Mix up with classical waves & 26 \\
& C2 & Mix up of well and barrier & 7 \\
Epistemological & C3 & Mix up with energy level & 31 \\
& E1 & Mix up with other classical concepts & 4 \\
& E2 & Use of inappropriate atomic models & 6 \\
\hline \hline
\end{tabular}

of the different types of learning impediments within the complete group of students. $14 \%$ of the explanations and open-ended questions were not answered, or answered by saying it was a guess.

As can be seen in Table VII, few students showed fragmentation learning impediments. This is partly due to the fact that students who could not make sense of a topic or question often did not explain their reasoning. Still, the three categories found in the subset were also present in the complete group of students. Furthermore, no other students were found within the compete group that had a linguistic learning impediment.

Pedagogic learning impediments were found more often. This type of learning impediment was difficult to discern, since both associated difficulties are implicit and result in other learning impediments. Difficulty caused by the mixed representation of the potential well and the wave function (Fig. 2) was assigned when students specifically linked the $y$ axis of the wave function to energy. Still, many students mixed up the amplitude and the energy level without specifically doing this, but this does not necessarily rule out a pedagogic learning impediment. This was also the case for knowledge of the exponential decrease of the wave function within the barrier, since many students had difficulty explaining tunneling and often just stated trivialities. In the cases where this impediment was observed, students tried to explain why the height and width did or did not influence tunneling probability.

Many students showed creative or epistemological learning impediments. The creative learning impediments were often found when students were interpreting the wave function; students mixed up the amplitude with energy, or described electrons as particles that vibrate or move like a wave. Mix ups with other (semi) classical concepts were mix ups with nuclear fusion and cell walls. The epistemological learning impediments were mainly found when students were reasoning about tunneling. Many students reasoned with distance, or stated that it would take more time or effort for a particle to go through a wider barrier. Also, many students reasoned that a certain amount of energy is needed, some because they reasoned that energy is lost, others because they reasoned that the particle's energy level needs to be higher than the barrier's energy level.

\section{E. Overview of observed synthetic models}

The observed learning impediments show that students have difficulty integrating QM in their prior knowledge. The observed fragmentation learning impediments and the second pedagogic learning impediments (P2) were based on missing knowledge. The other pedagogic, linguistic, creative, and epistemological learning impediments were expressed as incorrect models in which students had added the new concepts incorrectly to their existing framework. When looking at Table VII and Fig. 4, P1 and C3 are related learning impediments, which correspond to a similar incorrect model. Hence, there are 8 main synthetic models that were observed: (i) the potential well is a physical well, (ii) a mix up with classical waves, (iii) a mix up of the potential well and the barrier, (iv) a mix up with the energy level, (v) a mix up with other classical concepts, (vi) the use of inappropriate atomic models, (vii) deterministic reasoning in terms of movement, and (viii) classical reasoning in terms of energy. Figure 5 gives an overview of these synthetic models, together with a visual representation that is based on students' wording.

\section{CONCLUSIONS}

In this study we investigated secondary school students' understanding of potential wells and tunneling. In this section, we give an overview of the main results and draw conclusions based on these results. Additionally, we will describe the implications for researchers and teachers. 


\section{A. Students' understanding of potential wells and tunneling}

Analysis of the conceptual knowledge test shows that Dutch secondary school students experienced difficulties that were also reported for students at the undergraduate level; students mixed up classical and quantum models, and overgeneralized prior classical knowledge.

In the MC questions the students show two main difficulties: (i) $24 \%$ of the students believed that the amplitude or equilibrium of the wave function is related to the energy level, (ii) approximately 50\% of the students believed that energy was lost during tunneling. We also observed that students believed that there was a difference in the influence height of the barrier in comparison to the influence of the width of the barrier.

In a qualitative analysis of students' explanations and answers to the open-ended questions we found several underlying difficulties. Regarding the 1D infinite potential well and wave functions, the major difficulties were related to wave functions. While reasoning about the $1 \mathrm{D}$ infinite potential well, most of the students knew that this model represented a limited space in which a particle is contained. However, several students explained the model incorrectly, referring to semiclassical atomic models, and only a few students described the 1D infinite potential well model in terms of potential energy. Additionally, the interviews showed that several students did not know what the vertical lines in this model represented. These results show that students still have difficulty relating the 1D infinite potential well model to their prior knowledge of atomic models and potential energy. The latter can be explained by the fact that there is little emphasis on potential energy other than gravitational energy in the Dutch secondary school curriculum. While interpreting wave functions, students often showed creative learning impediments. About one-fourth of the students created a mixed-up model of the wave and particle behavior in which the particle vibrated or moved across a sinusoidal pathway. Over onethird of the students mixed up the amplitude or equilibrium of the wave function with the energy level. These mix ups show that students have difficulty integrating the waveparticle duality within their existing knowledge structures.

With respect to tunneling, students mainly showed epistemological learning impediments. Students often reasoned deterministically and stated that a particle needed more time, effort, or distance to tunnel through a wider barrier. Many students had difficulty reasoning with energy and stated that the particles' energy needed to be higher than the barrier's energy, or at least needed a minimum energy level. Students had difficulty integrating the concept of probability density in their deterministic thinking. Additionally, students lacked knowledge of the behavior of the wave function in a potential barrier, which made it difficult to reason about what happens with the energy and probability.
In retrospect, different learning impediments were found for potential wells and wave functions, and tunneling. The main learning impediment for potential wells and wave functions was creative, while for tunneling it was epistemological. This can be explained by the way QM is implemented in the Dutch secondary school curriculum. For the $1 \mathrm{D}$ infinite potential well, there is emphasis on both the energy representation and the wave function, and when students try to make sense of it, they try to integrate these two representations into one model. For tunneling, however, only the energy representation is used, causing students to connect this new topic only to their prior knowledge of energy or energy diagrams, the latter of which students have mainly seen while learning classical mechanics.

From the results of this study we can conclude that secondary school students have difficulty incorporating new knowledge of the 1D infinite potential well and tunneling into their thinking. Mix ups (creative learning impediments) arise mainly when students have to work with both energy and wave function representations, while the use of inappropriate classical reasoning (epistemological learning impediments) often occurs when students describe potential energy diagrams.

\section{B. Implications}

The research presented here shows that Dutch secondary school students have several difficulties in understanding the 1D infinite potential well and tunneling after being taught QM. The main problems were related to incorrect connections with prior knowledge. Some of the synthetic models found in this study are related to a lack of knowledge of the wave function, other synthetic models are related to students' inability to interpret potential energy diagrams. According to Vosniadou, Vamvakoussi, and Skopeliti [26], synthetic models can be avoided when instruction shows students how to connect their prior knowledge to the new concept. For students' understanding of QM, this could imply that teachers should support understanding by introducing the wave function for tunneling, and connect it to prior knowledge of the wave function, which students have already encountered in the context of the 1D infinite potential well. It could also imply that teachers should also make an effort to promote students' understanding of classical potential energy diagrams in order to improve their prior knowledge, and connect this to QM energy diagrams.

However, since QM is inconsistent with the classical models that students have learned, showing how to integrate QM with students' prior knowledge may not be so straightforward. At present, it is not clear what influence greater prior knowledge of underlying concepts, such as waves and potential energy, has on students' understanding of QM. Additionally, it is not clear to what extent students need to integrate QM within their prior knowledge in order to be able to decide which model is 
needed. Therefore, there is a need for more research into the influence of prior knowledge on students' understanding of QM, and into the prerequisites students need to be able to make an appropriate choice between representations.

\section{ACKNOWLEDGMENTS}

We thank the participating teachers for their time and effort. This work was funded by The Netherlands Organization for Scientific Research (NWO) under Grant No. 023.003.053.
[1] V. Karakostas and P. Hadzidaki, Realism vs. constructivism in contemporary physics: The impact of the debate on the understanding of quantum theory and its instructional process, Sci. Educ. 14, 607 (2005).

[2] E. K. Henriksen, B. Bungum, C. Angell, C. W. Tellefsen, T. Frågåt, and M. V. Bøe, Relativity, quantum physics and philosophy in the upper secondary curriculum: challenges, opportunities and proposed approaches, Phys. Educ. 49, 678 (2014).

[3] M. Ayene, J. Kriek, and B. Damtie, Wave-particle duality and uncertainty principle: Phenomenographic categories of description of tertiary physics students' depictions, Phys. Rev. ST Phys. Educ. Res. 7, 020113 (2011).

[4] C. Singh, M. Belloni, and W. Christian, Improving students' understanding of quantum mechanics, Phys. Today 59, No. 8, 43 (2006).

[5] H. K. E. Stadermann, E. van den Berg, and M. J. Goedhart, Analysis of secondary school quantum physics curricula of 15 different countries: Different perspectives on a challenging topic, Phys. Rev. Phys. Educ. Res. 15, 010130 (2019).

[6] K. Krijtenburg-Lewerissa, H. J. Pol, A. Brinkman, and W. R. v. Joolingen, Insights into teaching quantum mechanics in secondary and lower undergraduate education, Phys. Rev. Phys. Educ. Res. 13, 010109 (2017).

[7] A. Stefanel, M. Michelini, and L. Santi, Upper secondary school students learning pathways through quantum concepts, in Proceedings of the Esera 2011 Conference, Lyon, France (2011).

[8] D. Hoekzema, E. van den Berg, G. Schooten, and L. van Dijk, The particle/wave-in-a-box model in Dutch secondary schools, Phys. Educ. 42, 391 (2007).

[9] CVN, Nieuwe natuurkunde, advies-examenprogramma's voor havo en vwo (Nederlandse Natuurkundige Vereniging, Amsterdam, 2010).

[10] O. De Jong and K. S. Taber, The many faces of high school chemistry, in Handbook of Research in Science Education Volume II, edited by N. G. Lederman and S. K. Abell (Routledge, New York, 2014), p. 457.

[11] Ö. Özcan, N. Didiş, and M. F. Tasar, Students'conceptual difficulties in quantum mechanics: Potential well problems, Hacettepe Univ. J. Educ. 36, 169 (2009).

[12] S. B. McKagan, K. K. Perkins, and C. E. Wieman, Deeper look at student learning of quantum mechanics: The case of tunneling, Phys. Rev. ST Phys. Educ. Res. 4, 020103 (2008).

[13] M. N. Muniz and M. T. Oliver-Hoyo, Investigating quantum mechanical tunneling at the nanoscale via analogy: Development and assessment of a teaching tool for upper-division chemistry, J. Chem. Educ. 91, 1546 (2014).

[14] J. T. Morgan and M. C. Wittman, Examining the evolution of student ideas about quantum tunneling, AIP Conf. Proc. 818, 73 (2006).

[15] S. B. McKagan, K. K. Perkins, and C. E. Wieman, Design and validation of the quantum mechanics conceptual survey, Phys. Rev. ST Phys. Educ. Res. 6, 020121 (2010).

[16] R. V. Olsen, Introducing quantum mechanics in the upper secondary school: A study in Norway, Int. J. Sci. Educ. 24, 565 (2002).

[17] Ö. Özcan, Investigating students' mental models about the nature of light in different contexts, Eur. J. Phys. 36, 065042 (2015).

[18] C. Singh, Student understanding of quantum mechanics at the beginning of graduate instruction, Am. J. Phys. 76, 277 (2008).

[19] M. T. H. Chi, Three types of conceptual change: Belief revision, mental model transformation, and categorical shift, in Handbook of Research on Conceptual Change, edited by S. Vosniadou (Routledge, New York, 2008), p. 61 .

[20] C. Baily and N. D. Finkelstein, Ontological flexibility and the learning of quantum mechanics, arXiv:1409.8499.

[21] K. S. Taber, Shifting sands: A case study of conceptual development as competition between alternative conceptions, Int. J. Sci. Educ. 23, 731 (2001).

[22] E. F. Mortimer, Conceptual change or conceptual profile change?, Sci. Educ. 4, 267 (1995).

[23] J. R. Hoehn and N. D. Finkelstein, Students' flexible use of ontologies and the value of tentative reasoning: Examples of conceptual understanding in three canonical topics of quantum mechanics, Phys. Rev. Phys. Educ. Res. 14, 010122 (2018).

[24] A. Gupta, D. Hammer, and E. F. Redish, The case for dynamic models of learners' ontologies in physics, J. Learn. Sci. 19, 285 (2010).

[25] S. Vosniadou, Reframing the classical approach to conceptual change: Preconceptions, misconceptions and synthetic models, in Second International Handbook of Science Education, edited by B. J. Fraser, K. Tobin, and C. J. McRobbie (Springer Netherlands, Dordrecht, 2012), p. 119.

[26] S. Vosniadou, X. Vamvakoussi, and I. Skopeliti, The framework theory to the problem of conceptual change, in International Handbook of Research on Conceptual Change, edited by S. Vosniadou (Routledge, New York, 2009), p. 31. 
[27] D. P. Ausubel, The Acquisition and Retention of Knowledge: A Cognitive View (Kluwer, Dordrecht, 2000).

[28] K. S. Taber, The mismatch between assumed prior knowledge and the learner's conceptions: A typology of learning impediments, Educ. Stud. Math. 27, 159 (2001).

[29] K.S. Taber, Learning quanta: Barriers to stimulating transitions in student understanding of orbital ideas, Sci. Educ. 89, 94 (2005).

[30] J. Falk, Students' depictions of quantum mechanics: a contemporary review and some implications for research and teaching, Masters thesis, Uppsala University, 2004.
[31] See Supplemental Material at http://link.aps.org/ supplemental/10.1103/PhysRevPhysEducRes.16.010132 for the questions of the online test on potential wells and tunneling.

[32] M. C. Wittmann, J. T. Morgan, and L. Bao, Addressing student models of energy loss in quantum tunnelling, Eur. J. Phys. 26, 939 (2005).

[33] S. B. McKagan and C. E. Wieman, Exploring student understanding of energy through the quantum mechanics conceptual survey, AIP Conf. Proc. 818, 65 (2006). 